\title{
Do Positive Schizotypal Symptoms Predict False Perceptual Experiences in Nonclinical Populations?
}

\author{
Elias Tsakanikos, PhD,* and Phil Reed, PhD ${ }^{\dagger}$
}

\begin{abstract}
We examined whether positive schizotypy (i.e., reports of hallucinatory and delusional-like experiences) in nonclinical participants could predict false perceptual experiences during detection of fast-moving words beyond a possible response bias. The participants $(N=160)$ were assigned to one of two conditions: they were asked either to make presence/absence judgments (loose criterion) or to read aloud every detected word (strict criterion). Regression analysis showed that high levels of positive schizotypy predicted false alarms in the loose condition and false perceptions of words in the strict condition. The obtained effects were independent of detection accuracy, task order, impulsivity, and social desirability. We discuss the results in the context of information processing biases linked to the positive symptomatology of schizophrenia. Clinical and theoretical implications are also considered.
\end{abstract}

Key Words: Cognitive biases, delusions, false perceptual experiences, hallucinations, positive symptoms of schizophrenia, schizotypy.

( $J$ Nerv Ment Dis 2005;193: 809-812)

$\mathrm{H}$ allucinations and delusions, often referred to as positive psychotic symptoms (Crow, 1980), are experienced as reactions to actual events, have immediate impact on behavior, and appear to be beyond voluntary control (Chapman and Chapman, 1988; Slade and Bentall, 1988). The presence of such symptoms has been linked to certain cognitive biases. Patients with delusions require less information before reaching a conclusion than nondeluded patients (Dudley et al., 1997; Garety et al., 1991; Huq et al., 1988), although their general reasoning ability remains intact (Kemp et al., 1997). Such biases may have a causal role in the formation of unusual beliefs (Lawrence and Peters, 2004). Patients with hallucinations tend to make premature and erroneous judgments when asked to guess the meaning of perceptually ambiguous words (Heilbrun and Blum, 1984), and tend to

\footnotetext{
*Institute of Psychiatry, King's College, London, United Kingdom; and $\dagger$ Department of Psychology, University of Wales, Swansea, United Kingdom.

Send reprint requests to Elias Tsakanikos, $\mathrm{PhD}$, Institute of Psychiatry, King's College London, ESTIA Centre, 66 Snowfields, London SE1 3SS, United Kingdom. E-mail: etsakanikos@iop.kcl.ac.uk.

Copyright (C) 2005 by Lippincott Williams \& Wilkins

ISSN: 0022-3018/05/19312-0809

DOI: $10.1097 / 01 . n m d .0000188974 .44468 .92$
}

misattribute internally generated events to an external source (Brebion et al., 1998; Morrison and Haddock, 1997).

People who experience hallucinations also demonstrate a bias toward believing that a certain type of stimulus is present when it is actually absent (false alarms), although perceptual accuracy (correct responses) remains intact (Bentall and Slade, 1985; Cahill et al., 1996; but see Boecker et al., 2000). The same effect has been replicated in undergraduate students who score highly on measures of predisposition to hallucinations (Bentall and Slade, 1985; Rankin and O'Carroll, 1995). However, it remains unclear whether or not these participants actually had false perceptual experiences. An alternative possibility would be that such a bias might merely reflect an increased willingness to decide yes rather than no (i.e., adoption of a loose detection criterion or a positive response bias) when a binary response is required in a perceptually ambiguous situation.

Some researchers have suggested that cognitive biases are a main contributing factor to the maintenance, and possibly to the generation, of positive symptoms (Garety et al., 2001). Considering that cognitive-behavioral techniques seem promising in targeting psychotic symptoms (Gould et al., 2001; Pilling et al., 2002), modification of such biases could potentially contribute to clinical improvement. However, little is known about the principles that modulate these phenomena. For example, a tendency to see nonexisting events, as well as nonexisting relationships between events, is a common characteristic in many hallucinatory and delusional experiences. Such a tendency might constitute a crossmodal cognitive mechanism underlying the positive symptoms of schizophrenia. Yet it remains uncertain whether perceptually ambiguous conditions may lead someone not only to accept the presence of a certain stimulus in the absence of such a stimulus (i.e., false alarms due to a positive response bias) but also to describe in detail a nonexisting visual stimulus (i.e., false perceptions of events).

In this study, we employed a word detection task and examined whether positive schizotypal symptoms could predict, apart from a positive response bias, a bias to see nonexisting events. The main reason for studying such a bias as a function of symptoms of schizotypy in undergraduate students rather than schizophrenia patients was the utility of this approach in avoiding possible confounds (medication, hospitalization, and so forth) often inherent in research with clinical participants. Our main hypothesis was that positive schizotypal symptoms would predict false alarms, but not accuracy, replicating in the visual modality past evidence 
(Bentall and Slade, 1985; Cahill et al., 1996; Rankin and O'Carroll, 1995). In addition, we tested whether such bias would survive a strict response criterion (i.e., whether positive schizotypal symptoms would predict false perceptions of words).

\section{METHODS}

\section{Participants}

One hundred sixty undergraduate students (69 males and 91 females) took part in the study. The average age was 19.9 years, ranging from 18 to 24 years. At the time of testing, all the participants had normal or corrected-to-normal (i.e., wearing glasses or contact lenses) vision. None of the participants admitted psychiatric or neurological history or psychoactive medication use.

\section{Schizotypy Measures}

The Oxford-Liverpool Inventory for Feelings and Experiences (O-LIFE; Mason et al., 1995) consists of 159 items selected on the basis of factor-analytic studies of older schizotypy scales. The inventory includes four scales, following factor-analytic studies that have revealed three or four factors underlying the construct of schizotypy (Bentall et al., 1989; Vollema and van den Bosch, 1995). The first three scales correspond to a three-factor model of schizophrenia (Liddle, 1987): positive (Unusual Experiences: 30 items), negative (Introvertive Anhedonia: 27 items), and disorganized (Cognitive Disorganization: 24 items). The total score for each scale is based on the endorsement of items (yes/no answers). The positive schizotypy scale consists of items assessing mainly atypical beliefs and unusual perceptual experiences. The fourth scale (Impulsive Nonconformity: 23 items) refers to the impulsive, aggressive, and asocial aspects of psychosis, and is largely based on Eysenck's Psychoticism scale (Eysenck and Eysenck, 1975). The Impulsive Nonconformity scale was included in the present analysis to evaluate the possibility of an increased false alarm rate as the result of impulsive tendencies often associated with psychotic-like characteristics. The inventory also included the Lie scale of the Eysenck Personality Questionnaire (Eysenck and Eysenck, 1975), which was used to assess whether decision biases would be related to a bias to respond in a socially desirable way to please the experimenter.

Based on previous studies (e.g., Tsakanikos, 2004; Tsakanikos and Reed, 2003), the typical range of scores on the O-LIFE scales is as follows: Unusual Experiences, 0-27; Cognitive Disorganization, 0-22; Introvertive Anhedonia, 0-15; and Impulsivity Nonconformity, 0-19.

\section{Stimuli and Apparatus}

Each participant received 64 trials as a continuous sequence of short animated images. Half of the trials contained a word among nonwords (word trials) and half of them contained only nonwords (nonword trials). Each trial depicted a display of four round blocks, one in each quadrant of the computer screen, which were identical in size to one another. The screen background was black, the blocks were gray, and the letters were white. In each block, there was either a nonword or a real word. The words were five-letter concrete nouns matched for frequency of occurrence. The nonwords were meaningless strings of five consonants, such as "GNFDW," "WJDTX," and so forth.

The animations were constructed on a three-dimensional model package (3-Dimensional Studio) and were presented with a multimedia animator player (Autodesk Animator Player for Windows, Version 1.0). The animations produced an impression of motion, such that the four-block configuration appeared to loom from a distance toward the observer. Each animation was composed of 74 frames and was presented at a rate of 9 frames/s.

\section{Procedure}

The participants were told that they would be taking part in a word detection task and that they would receive both word and nonword trials. The experimental procedure was a between-participant design. The participants were randomly assigned into two groups and were tested in individual cubicles equipped with PC monitors. In the loose condition $(N=$ 80), the participants were instructed to report yes when they saw a real word (word trials), and to report no when they did not see a real word (nonword trials). In the strict condition $(N=80)$, they were asked to read aloud any real word they could see and ignore the nonwords. Within each condition, half of the participants received, at the outset, the schizotypy inventory and then the detection task. The other half received the tasks in the opposite order. The responses were recorded by the experimenter. There was a time restriction in responding, as the participants were required to respond during the speeded presentation of the words. Accuracy (yes responses during the word trials in the loose condition and correct reports of words in the strict condition), false alarms (yes responses during nonword trials in the loose condition), and false perceptions (detailed descriptions of words during nonword trials in the strict condition) were the dependent variables. At the time of testing, the experimenter was unaware of the schizotypy level of the participants. Detailed information about the purpose of the study was given after the end of the session.

\section{RESULTS}

We employed the Statistical Package for Social Sciences (SPSS edition 11.1) for all the subsequent analyses. Table 1 shows the means, $S D$ s, and intercorrelations between the O-LIFE scales. The means and the SDs, as well as the pattern of intercorrelations, were comparable to these reported in the original study on the development of the scales

TABLE 1. Means and SDs of the Schizotypy Scales (O-LIFE), and Their Intercorrelations

\begin{tabular}{|c|c|c|c|c|c|c|}
\hline Schizotypy Scale & $M$ & $S D$ & Range & 1 & 2 & 3 \\
\hline 1. Unusual Experiences & 9.2 & 5.76 & 28 & - & & \\
\hline 2. Cognitive Disorganization & 12.23 & 5.93 & 23 & $0.36^{*}$ & - & \\
\hline 3. Introvertive Anhedonia & 4.18 & 3.39 & 14 & 0.03 & $0.28 *$ & - \\
\hline 4. Impulsive Nonconformity & 10.04 & 3.78 & 19 & $0.33 *$ & $0.31 *$ & 0.05 \\
\hline
\end{tabular}


(Mason et al., 1995) as well as to these reported in later studies (e.g., Tsakanikos, 2004; Tsakanikos and Reed, 2003).

Preliminary inspection of the detection data revealed that the distributions were negatively skewed (skewness values $>4$ times their $S E$ s); therefore, they were logarithmically transformed by employing the Blom estimation formula $([\mathrm{r}-3 / 8] /[\mathrm{w}+1 / 4]$, where $r=\operatorname{rank}$ and $w=$ sum of the case weights).

In the loose condition, accuracy (mean $z$ score $=-.18$; $S D=.72)$ did not correlate significantly $(r=.02 ; p>0.30)$ with false alarms (mean $z$ score $=.31 ; S D=.68$ ), suggesting the absence of a trade-off between accuracy and false alarms. Likewise, in the strict condition, accuracy (mean $z$ score $=$ $.17 ; S D=1.16)$ did not correlate significantly $(r=-.04$; $p>0.30$ ) with false perceptions of words (mean $z$ score $=$ $-.25 ; S D=1.01)$. In both conditions, the effect of task on the dependent measures was not statistically significant ( $t$ values $<1)$. The correlation between social desirability and the dependent measures was not statistically significant ( $p$ values $>0.30$ ). Accuracy was significantly higher in the loose condition than in the strict condition $\left(t_{(158)}=-2.31 ; p<0.05\right)$. Correspondingly, erroneous responses were significantly lower in the strict than in the loose condition $\left(t_{(158)}=-4.15\right.$; $p<0.01)$. Erroneous responses correlated significantly only with positive schizotypy in both strict $(r=.32 ; p<0.005)$ and loose $(r=.31 ; p<0.005)$ condition. No other correlation between schizotypy measures and performance parameters was statistically significant ( $p$ values $>0.20)$.

To examine whether schizotypy scores could predict false alarms in the loose and in the strict condition, while controlling for the intercorrelations, we performed a set of simultaneous multiple regression analyses (method: enter). In the first regression analysis, the scores on the four O-LIFE scales were the predictor variables, and the $z$ scores of incorrect yes responses (false alarms) obtained in the loose condition were the dependent variable. The overall equation was statistically significant $\left(F_{(4,79)}=2.62 ; p<0.05\right)$, accounting for about $8 \%$ (adjusted $R^{2}$ ) of the variance. However, positive schizotypy (Unusual Experiences) was the only significant predictor $(\beta=.37 ; t=2.97 ; p<0.01)$. In the second regression analysis, the O-LIFE scales were the predictor variables, and the $z$ scores of the erroneous responses (i.e., false perceptions of words) obtained in the strict condition during nonword trials were the dependent variable. The regression equation was statistically significant $\left(F_{(4,79)}=\right.$ $3.31 ; p<0.01)$, accounting for about $11 \%$ of the variance (adjusted $R^{2}$ ). As in the previous analysis, positive schizotypy (Unusual Experiences) was the only significant individual predictor $(\beta=.40 ; t=3.42 ; p<0.001)$. A third regression analysis with erroneous responses during word trials as dependent measure failed to reach statistical significance $(F<$ $1)$. The results of these regression analyses are summarized in Table 2.

To examine whether scores on the schizotypy scales could predict accuracy, we performed two further multiple regressions (one for each condition) with the O-LIFE scales as predictor variables. Neither for the loose $\left(F_{(4,79)}=1.11\right.$; $p>0.30)$ nor for the strict condition $(F<1)$ were the overall
TABLE 2. O-LIFE Scores as Predictor Variables for Erroneous Responses in Loose and Strict Condition

\begin{tabular}{lrrrr}
\hline & B & $\boldsymbol{S E B}$ & $\boldsymbol{\beta}$ & \multicolumn{1}{c}{$\boldsymbol{t}$} \\
\hline Loose condition & & & & \\
$\quad$ Unusual Experiences & 0.05 & 0.02 & 0.37 & $2.97^{*}$ \\
Cognitive Disorganization & 0.01 & 0.01 & 0.02 & 0.15 \\
$\quad$ Introvertive Anhedonia & -0.01 & 0.02 & -0.06 & -0.62 \\
$\quad$ Impulsive Nonconformity & -0.02 & 0.02 & -0.13 & -1.12 \\
$\quad$ Constant & 0.16 & 0.22 & & \\
Strict condition & & & & \\
$\quad$ Unusual Experiences & 0.06 & 0.02 & 0.39 & $3.42^{* *}$ \\
$\quad$ Cognitive Disorganization & -0.02 & 0.02 & -0.09 & -0.79 \\
$\quad$ Introvertive Anhedonia & 0.04 & 0.03 & 0.14 & 1.26 \\
Impulsive Nonconformity & -0.05 & 0.03 & -0.17 & -1.48 \\
Constant & -0.33 & 0.38 & & \\
\hline
\end{tabular}

$* p<0.01$ (two-tailed); $* * p<0.001$ (two-tailed).

regression equations significant. In neither of these analyses was there any significant individual predictor (smaller $p>$ $0.20)$.

\section{General Discussion}

During visual detection of fast-moving words, undergraduate students scoring highly on positive schizotypy reported seeing words that did not exist in the list. When the task required a yes (target presence) or no (target absence) response, high schizotypy scorers demonstrated a positive response bias, although their accuracy remained intact. These findings replicate and extend past evidence, in the visual modality, that hallucinations, as well as psychometrically defined proneness to hallucinations, are linked to a bias toward believing that an auditory stimulus is present in the absence of such a stimulus (Bentall and Slade, 1985; Rankin and O'Carroll, 1995). Furthermore, when the task required a detailed description of the target (strict condition), positive schizotypal symptoms predicted false perceptions of words.

In the strict condition, all the erroneous responses during nonword trials were new words. In the same condition, further analysis revealed that positive schizotypy did not predict erroneous responses during word trials. This is consistent with the finding that patients with hallucinations during verbal transformation tasks report hearing new words, although patients without hallucinations report only verbal transformations of existing words (Haddock et al., 1995).

Normal perception seems to entail fundamental decision-making processes (Nakayama, 2001). Similar top-down mechanisms are also central to many recent theoretical models of hallucinations (e.g., Behrendt, 1998; Boecker et al., 2000; Grossberg, 2000). A relevant cognitive mechanism might constitute a biased attributional process, possibly activated when dealing with an ambiguous situation. According to the externality hypothesis (Garety et al., 2001), schizophrenic patients are inclined to accept the possibility that an experienced event (for example, a "voice") is an externally generated event rather than an internally generated input. 
The externality hypothesis can also explain false perceptual experiences in high schizotypy scorers during the presently employed task. At an early stage, simultaneous presentation of fast moving nonwords may have generated past associations or verbal representations of corresponding words on the basis of some superficial similarity. Although such generated events could have occurred for every participant regardless of their schizotypy status, high-schizotypy scorers tend to produce proportionately more uncommon verbal associations on word-association tasks (Miller and Chapman, 1983). A cognitive experience of this type could be interpreted either as an internally generated event ("I just saw something that looked like the word ZEBRA, but this word is not actually there"-rejection of the externality hypothesis) or as an externally generated event ("I just saw the word ZEBRA"- acceptance of the externality hypothesis). As a consequence of such an attributional bias, it is possible that high positive schizotypy scorers were more likely to "translate" their internally generated experiences (i.e., associations and verbal representations of similar-looking/sounding words) into perceptual experiences (i.e., false perceptions of words).

An alternative explanation could be that the present findings might reflect a data-gathering bias (a jumping-toconclusions type bias) where fewer data are required before reaching a conclusion (Dudley et al., 1997; Garety et al., 1991; Kemp et al., 1997). Such an explanation seems less likely because a bias of this type would also influence other aspects of performance, but this was not the case. A datagathering bias would also be expected to affect erroneous responses during word trials; however, positive schizotypy was associated with erroneous responses only in nonword trials.

The obtained findings have both clinical and theoretical implications. Further support is provided to the notion that psychotic-like experiences do not need to involve aberrant cognitive processes. On average, each participant saw one to two words that never appeared in the trials; however, participants scoring highly on positive schizotypy were more inclined to see such words. Similarly, psychotic-like perceptual experiences are likely to be exaggerations of processes modulating normal perception. In addition, a bias to see nonexisting events, as assessed in word-detection paradigms, could be developed into a behavioral outcome measure, complementary to clinical assessment and rating scales, for evaluating clinical interventions. Therefore, longitudinal studies to evaluate whether or not such biases could predict the onset of clinical symptoms would be particularly informative.

In summary, we found that positive schizotypal symptoms, as assessed psychometrically in nonclinical young adults, predicted false perceptions in the laboratory. Understanding the mechanisms underlying such phenomena may contribute not only to the development of effective cognitivebehavioral interventions for positive symptoms of schizophrenia but also to early detection and prevention.

\section{ACKNOWLEDGMENTS}

Thanks are due Michael Ciarleglio, Helena Drury, Mayko Fukui, and Lee Jones for their help with the data collection.

\section{REFERENCES}

Bentall RP, Claridge GS, Slade PD (1989) The multidimensional nature of schizotypal traits: A factor analytic study with normal subjects. Br J Clin Psychol. 28:363-375.

Bentall RP, Slade PD (1985) Reality testing and auditory hallucinations: a signal detection analysis. Br J Clin Psychol. 24:159-169.

Behrendt RP (1998) Under-constrained perception: A theoretical approach to the nature and function of verbal hallucinations. Compr Psychiatry. 39:236-248

Boecker KBE, Hijman R, Kahn RS, De Haan EHF (2000) Perception, mental imagery and reality discrimination in hallucinating and non-hallucinating schizophrenic patients. Br J Clin Psychol. 39:397-406.

Brebion G, Smith MJ, Amador X, Malaspina D, Gorman JM (1998) Word recognition, discrimination accuracy and decision bias in schizophrenia: Associations with positive symptomatology and depressive symptomatology. J Nerv Ment Dis. 186:604-609.

Cahill C, Silbersweig D, Frith C (1996) Psychotic experiences induced in deluded patients using distorted auditory feedback. Cogn Neuropsychiatry. 1:201-211.

Chapman LJ, Chapman JP (1988) The genesis of delusions. In TF Oltmanns, BA Maher (Eds), Delusional Beliefs. New York: John Wiley \& Sons.

Crow TJ (1980) Molecular pathology of schizophrenia: More than one disease process. BMJ. 280:66-68.

Dudley REJ, John CH, Young AW, Over DE (1997) Normal and abnormal reasoning in people with delusions. Br J Clin Psychol. 36:243-258.

Eysenck HJ, Eysenck SBG (1975) Manual of the Eysenck Personality Questionnaire. London: Hodder and Stoughton.

Garety PA, Hemsley DR, Wessely S (1991) Reasoning in deluded schizophrenic and paranoid patients: Biases in performance on a probabilistic inference task. J Nerv Ment Dis. 179:194-201.

Garety PA, Kuipers E, Fowler D, Freeman D, Bebbington PE (2001) A cognitive model of the positive symptoms of psychosis. Psychol Med. 31:189-195.

Gould RA, Mueser KT, Bolton E, Mays V, Goff D (2001) Cognitive therapy for psychosis in schizophrenia: An effect size analysis. Schizophr Res. 48:335-342.

Grossberg S (2000) How hallucinations may arise from brain mechanisms of learning, attention and volition. J Int Neuropsychol Soc. 6:583-592.

Haddock G, Slade PD, Bentall RP (1995) Auditory hallucinations and the verbal transformation effect. Pers Individ Diff. 19:301-306.

Heilbrun AB, Blum NA (1984) Cognitive vulnerability to auditory hallucination: Impaired perception of meaning. Br J Psychiatry. 144:508-512.

Huq SF, Garety PA, Hemsley DR (1988) Probabilistic judgements in deluded and non-deluded subjects. $Q J$ Exp Psychol A. 40:801-812.

Kemp R, Chua S, McKenna P, David A (1997) Reasoning and delusions. Br J Psychiatry. 170:398-405.

Lawrence E, Peters E (2004) Reasoning in believers in the paranormal. J Nerv Ment Dis. 192:727-733.

Liddle P (1987) The symptoms of chronic schizophrenia: A re-examination of the positive-negative dichotomy. Br J Psychiatry. 151:221-234.

Mason O, Claridge G, Jackson M (1995) New scales for the assessment of schizotypy. Pers Individ Diff. 53:727-730.

Morrison AP, Haddock G (1997) Cognitive factors in source monitoring and auditory hallucinations. Psychol Med. 27:669-679.

Miller EN, Chapman LJ (1983) Continued word association in hypothetically psychosis-prone college students. J Abnorm Psychol. 92:468-478.

Nakayama K (2001) Modularity in perception, its relation to cognition and knowledge. In EB Goldstein (Ed), Blackwell Handbook of Perception. Malden (MA): Blackwell Publishers.

Pilling S, Bebbington P, Kuipers E, Garety P, Geddes J, Orbach G, Morgan C (2002) Psychological treatments in schizophrenia, I: Meta-analysis of family intervention and cognitive behaviour therapy. Psychol Med. 32:763-782.

Rankin PM, O’Carroll PJ (1995) Reality discrimination, reality monitoring and disposition towards hallucination. Br J Clin Psychol. 34:517-528.

Slade PD, Bentall RP (1988) Sensory Deception: A Scientific Analysis of Hallucination. Baltimore (MD): Johns Hopkins University Press.

Tsakanikos E, Reed P (2003) Visuo-spatial processing and dimensions of schizotypy: Figure-ground segregation as a function of psychotic-like features. Pers Individ Diff. 35:703-712.

Tsakanikos E (2004) Logical reasoning in schizotypal personality. Pers Individ Diff. 37:1717-1726.

Vollema MG, van den Bosch RJ (1995) The multidimensionality of schizotypy. Schizophr Bull. 21:19-31. 